\title{
Dynamic Modeling and Stochastic Prediction of the Robot's Soccer Ball Trajectory
}

\author{
Aline R. Silva * Leonardo R. Olivi* Exuperry B. Costa* \\ Ana Sophia C. A. Vilas Boas* \\ * Faculdade de Engenharia Elétrica, Universidade Federal de Juiz de \\ Fora, MG, (e-mail: aline.rocha@engenharia.ufjf.br).
}

\begin{abstract}
Robot soccer is a dynamic, versatile and real-time execution environment, so the knowledge of the position of the elements of the game in the field, such as robots, ball, field lines, goals, among others, becomes essential to have play and reach the goal goal. In this context, tracking the ball is an important tool to know the position of the ball in the field and predictions of future positions contributes to the efficiency of the robot in its trajectory of chasing the ball. In the present work the tracking of the ball was developed through the Kalman Filter, by means of the modeling of the equations that represent the position and speed of the ball, as well as its validation. The results show that the method developed in this work obtains accurate predictions, amplifying the strategies for the game.

Resumo: O futebol de robôs é um ambiente dinâmico, versátil e de execução em tempo real, logo, o conhecimento da posição dos elementos do jogo em campo, como robôs, bola, linhas de campo, gols, entre outros, se faz essencial para se ter domínio do jogo e atingir o objetivo de fazer o gol. Nesse contexto, o rastreamento da bola é uma importante ferramenta para se conhecer a posição da mesma no campo e previsões de posições futuras contribui na eficiência do robô na trajetória do mesmo de perseguir a bola. No presente trabalho desenvolveu-se o rastreamento da bola através do Filtro de Kalman, por meio da modelagem das equações que representam a posição e velocidade da bola, bem como, a validação das mesmas através de simulações. Os resultados mostram que o método desenvolvido neste trabalho obtém predições acuradas, ampliando as estratégias de jogo.
\end{abstract}

Keywords: Robots Football; Dynamic Modeling; Extended Kalman Filter; Trajectory tracking; Stochastic Prediction.

Palavras-chaves: Futebol de Robôs; Modelagem Dinâmica; Filtro de Kalman Estendido; Rastreamento de trajetória; Predição Estocástica.

\section{INTRODUÇÃO}

Um sistema de rastreamento pode ser usado para observação, monitoramento e predição de dados em relação a movimentação de um objeto de estudo, com a capacidade de fornecer elementos para um processamento posterior e realizar tomadas de decisões baseadas nas informações obtidas e rastreadas. Nesse sentido, um sistema robusto de rastreamento pode prover informações sobre o movimento relativo de um objeto em relação ao observador e pode simplificar a obtenção da forma ou localização de um objeto (Motta and Tourino, 2008).

Ao longo do tempo, diversas técnicas de rastreamento foram desenvolvidas com grande relevância, destacandose os Filtros Bayesianos, e empregados em áreas multidisciplinares como robótica, segurança, logística, biomédica, prestação de serviços, entre outras. Dentre essas, destacam-se aplicações como rastreamento de pessoas e veículos (Montanari, 2015), sistema de vigilância visual, monitoramento de tráfego, realidade virtual, análise de movimentos humanos (Rivero, 2004), análise de desempenho de atletas e muitas outras aplicações.

A captação de informações pelos métodos de rastreamento provém de dados obtidos pelo uso de sensores tais como câmeras, LIDAR, laser, GPS, ultrassônico. Em aplicações de robótica autônoma, tarefas fundamentais a serem realizadas são mapeamento e/ou reconhecimento de um cenário/ambiente, localização, detecção e rastreamento de objetos em movimento e planejamento de movimento (Amaral, 2015). Nesse contexto, as competições de robótica abrangem essas tarefas e são ferramentas para desenvolvimento e/ou utilização das técnicas de rastreamento.

O futebol de robôs é um dos ramos das competições de robótica autônoma e representa um ambiente dinâmico e de tempo real, no qual o rastreamento de elementos do jogo, como robôs e bola, se mostram eficazes no que diz respeito aos robôs terem um maior conhecimento do ambiente em um dado momento possibilitando-os de agirem de forma mais rápida e assertiva e colaborando na elaboração de uma estratégia de jogo. 
Com o intuito de ampliar as possibilidades e estratégias do jogo, o presente trabalho tem por objetivo rastrear o comportamento da bola de futebol com o intuito de prever a trajetória deste elemento do jogo. O método é baseado nos dados obtidos pela câmera do robô e na utilização do Filtro de Kalman (FK) como ferramenta de predição e correção dos dados ruidosos. A categoria SPL (Standard Platform League) é o objeto de estudo, entretanto, a modelagem descrita é generalista, aplicável às demais categorias.

\section{REFERÊNCIA BIBLIOGRÁFICA}

Para a implementação do sistema de rastreamento da bola foram pesquisados e analisados na literatura os métodos mais recorrentes para o rastreamento de objetos. Após a análise, o estudo concentrou-se no método do Filtro de Kalman, nas representações físicas do comportamento da bola em campo e na eficácia ao modelo dinâmico do jogo.

As técnicas de rastreamento se dividem em três principais. A primeira é baseada nos métodos de regressão e predição clássicas, com modelos de autorregressão (AR), com variável exógena (ARX), modelo ARMAX abordado por (Simões et al., 2002). A segunda variante na literatura é o uso das redes neurais artificiais para prever a trajetória da bola discutida por (Botta et al., 2013). A terceira técnica é o Filtro de Kalman, sendo a mais utilizada por diversas categorias do futebol de robôs, entre as implementações encontradas estão a abordagem dada por (Malheiro, 2010) na categoria SSL e pela categoria SPL se destacam os estudos realizados pelas equipes B-Human da Alemanha (Röfer et al., 2017) e UNSW da Austrália (Teh, 2011).

Como o Filtro de Kalman necessita de uma modelagem do sistema rastreado, no caso a trajetória da bola, na literatura foram encontradas três formas diferentes de modelar o movimento do objeto em estudo. A primeira forma de modelagem é conforme discutida em (Teh, 2011) e (Junior, 2015), considerando o movimento retilíneo uniformemente variado da Física Clássica. A segunda modelagem é discutida em (Teh, 2011), considerou-se um modelo de desaceleração linear, no qual a posição atual é dependente apenas da posição anterior e da velocidade no tempo e a velocidade atual apenas decai de $10 \%$ da velocidade anterior. A terceira modelagem é abordada por (Bustamante et al., 2007), este descreve a trajetória baseado nas forças atuantes na bola durante o jogo.

\section{DEFINIÇÕES TÉCNICAS}

Como ponto de partida do estudo foi realizada uma análise do comportamento da bola com a finalidade de conhecer o tipo de trajetória percorrida pela mesma no campo de jogo. Para isso, utilizou-se o robô NAO chutando a bola oficial no campo de carpete e filmou-se o chute com uma câmera posicionada acima do campo, conforme Figura 1.

O vídeo obtido foi analisado com o software Tracker e a trajetória percorrida pela bola no plano X-Y pode ser visualizada na Figura 1, assim como o perfil de trajetória ao longo dos eixos $x$ e $y$ em relação ao tempo pode ser visualizado na Figura 2.

Pela observação do teste e pela análise obtida no software de processamento de imagens, pode-se verificar que a

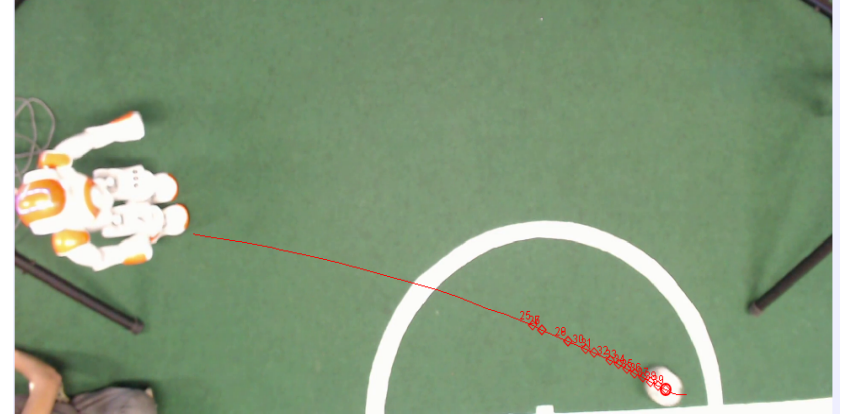

Figura 1. Trajetória da bola após chute do NAO.

bola tende a fazer uma trajetória retilínea. Entretanto, como existem erros inerentes a movimentação da mesma, imperfeições no solo e no carpete, estes fatores fazem a bola ter um desvio do seu comportamento natural.

Além disso, foi analisado que a movimentação da bola é bidimensional, ou seja, limitada ao plano X-Y (2D), assim, a bola não é rebatida na direção do eixo $z$, o que poderia caracterizar uma altura no deslocamento da bola e a dinâmica necessitaria ser tridimensional (3D).

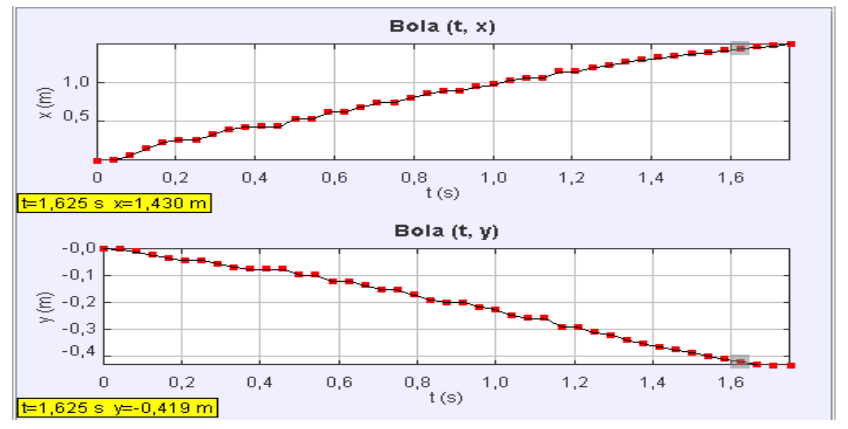

Figura 2. Trajetória da bola ao longo do tempo.

Os testes demonstraram ainda que o ângulo da bola com relação ao referencial X-Y do campo é definido pela angulação do robô em campo e representado matematicamente por $\theta$. E ainda pode existir uma angulação entre o pé do robô e o plano X-Y do campo, essa angulação pode interferir na força do chute da bola, sendo representado matematicamente por $\phi$, ainda que para o caso do robô $\mathrm{NAO}$ o $\phi$ é praticamente zero pelo formato do pé do robô.

O sistema de coordenadas global é definido conforme mostrado na Figura 3. O centro do campo é definido como

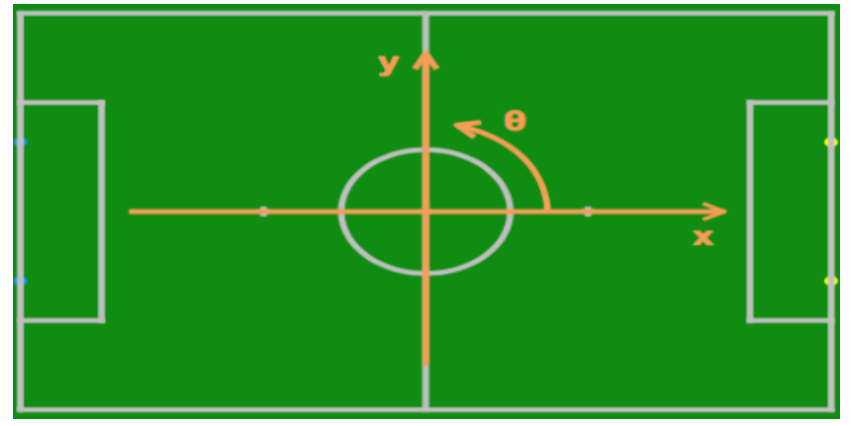

Figura 3. Sistema de coordenadas global

a origem, o eixo X se estende na linha horizontal, o sentido de crescimento é da esquerda para a direita, o eixo Y é a 
linha vertical perpendicular ao $\mathrm{X}$ e o $\mathrm{Z}$ é orientado para cima. $\mathrm{O}$ ângulo $\theta$ é marcado como zero no eixo $x$ e cresce no sentido anti-horário (regra da mão direita).

\section{FUNDAMENTAÇÃO MATEMÁTICA}

Analisando a física que envolve o sistema NAO, campo e bola, propõe-se um modelo matemático para predizer as posições e velocidades da bola durante o jogo.

\subsection{Definição da movimentação}

Um modelo do movimento de um objeto dinâmico é descrito como uma função que varia nas relações de posição e velocidade atuais $\left(p_{t}, v_{t}\right)$, no tempo $t$, até um futuro $\left(p_{t+i}, v_{t+i}\right)$ no tempo $t+i$ (Bustamante et al., 2007).

Tendo isto em mãos, tem-se, formalmente, que:

$$
M:\left(p_{t}, v_{t}\right) \rightarrow\left(p_{t+i}, v_{t+i}\right) \text {. }
$$

Em que:

$p$ é o vetor posição;

$v$ é o vetor velocidade;

$i$ é a iteração em que $i>0$ é uma condição. Ainda é possível definir em termos da mobilidade bidimensional da bola. Logo, a posição e a velocidade podem ser definidas como os vetores mostrados nas Equações (2) e (3), respectivamente,

$$
\begin{aligned}
& p_{t}=\left\langle p_{x}, p_{y}\right\rangle \\
& v_{t}=\left\langle v_{x}, v_{y}\right\rangle .
\end{aligned}
$$

\subsection{Definição do sistema de forças}

No sistema de forças que envolve o NAO, o campo e a bola, conforme caracterizado na Figura 4, as forças atuantes na bola e que imprimem movimentação e desaceleração na mesma são as forças do chute do NAO e a de desaceleração da bola, enumeradas a seguir:

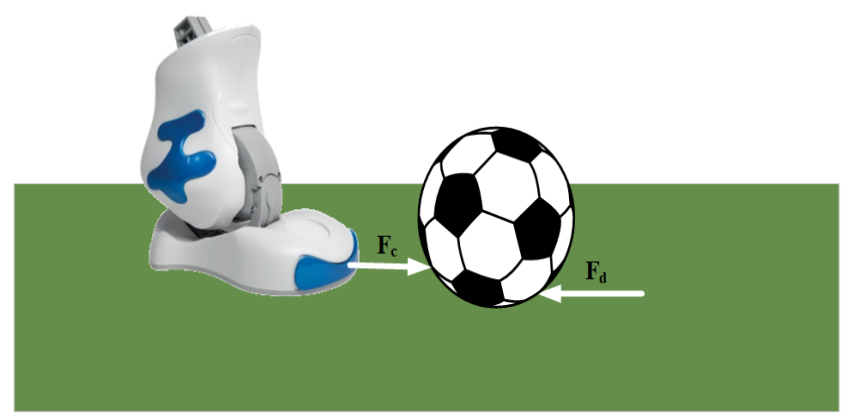

Figura 4. Forças atuantes no sistema NAO, campo e bola

(1) A força do chute do NAO: a aceleração da bola se dá sempre que um NAO a chuta ou a conduz com toques do pé. O ato de conduzir a bola pode ser caracterizado como um chute com menor potência. Neste trabalho, essa força impressa pelos atuadores do pé do NAO é chamada de força do chute, simbolizada por $F_{c}$.

A força do chute é um vetor definido como:

$$
\boldsymbol{F}_{\boldsymbol{c}}=\left\langle F_{c x}, F_{c y}\right\rangle \text {. }
$$

Durante a fase de testes e ensaios deste trabalho, observou-se que a intensidade dessa força é constante durante o tempo de contato do robô com a bola, passado esse contato a força continua sua ação sob a bola, entretanto sua intensidade diminui gradativamente até que a força resultante que atua na bola pare o seu movimento. Logo, para caracterizar a força do chute foi escolhida a função $G B$ ell, representada pela Equação (5), podendo ser visualizada na Figura 5,

$$
\left|F_{c}\right|=\frac{1}{1+\left|\frac{t-c}{a}\right|^{2 b}} \text {. }
$$

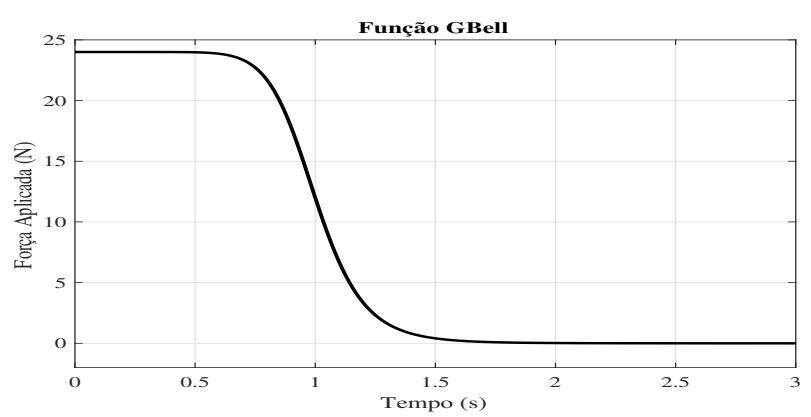

Figura 5. Representação da Força do chute com a função GBell

Considerando que possa existir uma angulação $\phi_{c}$ entre o pé do robô e o plano do campo, e ainda uma angulação $\theta_{c}$ entre o chute e a bola, tem-se que:

$$
\begin{gathered}
F_{c x}=F_{c} \cos \left(\phi_{c}\right) \cos \left(\theta_{c}\right), \\
F_{c y}=F_{c} \cos \left(\phi_{c}\right) \sin \left(\theta_{c}\right) .
\end{gathered}
$$

(2) Força de desaceleração: força que atua diminuindo a velocidade da bola e é contrária ao movimento do objeto, sua ação perdura até a resultante da força que atua na bola seja zero e cesse o movimento, é caracterizada pela força de atrito com o solo e simbolizada por $F_{d}$.

A força de desaceleração pode ser definida como:

$$
F_{d}=-\xi_{B} v \text {. }
$$

No qual $\xi_{B}$ é a constante que representa a desaceleração da bola e depende do corpo em que a força atua.

\subsection{Modelagem dinâmica da bola}

Este trabalho considerará uma visão mecanicista para a modelagem da bola. Portanto, de acordo com a Segunda Lei de Newton: "A força resultante que atua sobre um corpo é proporcional ao produto da massa pela aceleração por ele adquirida" (Garcia, 2005). Assim, a afirmação anterior pode ser resumida nas Equações (9), (10) e (11) para o caso da bola, no qual o somatório da força do chute do robô com a força de desaceleração modelará o comportamento da bola em campo durante o jogo,

$$
\sum F=m_{B} a \text {. }
$$

E,

$$
\sum F=F_{c}-F_{d}
$$

Logo,

$$
\sum F=F_{c}-\xi_{B} v .
$$

Em que:

$m_{B}$ : massa da bola; 
$a$ : aceleração da bola;

$F$ : força resultante na bola;

$v$ : velocidade da bola.

Considerando as componentes das forças e a decomposição da força do chute no plano X-Y, as equações que regem a movimentação da bola para cada eixo são dadas por:

$$
\begin{gathered}
\sum F_{x}=m_{B} a_{x}, \\
\sum F_{y}=m_{B} a_{y}, \\
\sum F_{x}=F_{c} \cos \left(\phi_{c}\right) \cos \left(\theta_{c}\right)-\xi_{B} v_{x}=m_{B} a_{x}, \\
\sum F_{y}=F_{c} \cos \left(\phi_{c}\right) \sin \left(\theta_{c}\right)-\xi_{B} v_{y}=m_{B} a_{y} .
\end{gathered}
$$

Em que:

$F_{x}$ : força resultante na direção de $x$ da bola;

$F_{y}$ : força resultante na direção de $y$ da bola;

$a_{x}$ : aceleração na direção de $x$ da bola;

$a_{y}$ : aceleração na direção de $y$ da bola;

$v_{x}$ : velocidade na direção de $x$ da bola;

$v_{y}$ : velocidade na direção de $y$ da bola.

As equações diferencias que regem o deslocamento da bola são dadas por:

$$
\begin{aligned}
& m_{B} \frac{\mathrm{d} v_{x}}{\mathrm{~d} t}+\xi_{B} v_{x}=F_{c x} \\
& m_{B} \frac{\mathrm{d} v_{y}}{\mathrm{~d} t}+\xi_{B} v_{y}=F_{c y}
\end{aligned}
$$

Em que:

$$
\begin{aligned}
F_{c x} & =F_{c} \cos \left(\phi_{c}\right) \cos \left(\theta_{c}\right), \\
F_{c y} & =F_{c} \cos \left(\phi_{c}\right) \sin \left(\theta_{c}\right) .
\end{aligned}
$$

Para resolver a equação diferencial pode-se considerar a EDO genérica para $\mathrm{X}$ e $\mathrm{Y}$ :

$$
\frac{\mathrm{d} v}{\mathrm{~d} t}+\frac{\xi_{B}}{m_{B}} v=\frac{F_{c}}{m_{B}}
$$

Dessa forma, resolvendo a equação diferencial, obtém-se como solução:

$$
v(t)=A e^{\left(-\frac{\xi_{B}}{m_{B}}\right) t}+\frac{F_{c}}{\xi_{B}} .
$$

A constante $A$ pode ser determinada pela condição inicial do sistema, para cada tempo $t$ tem-se uma velocidade associada, logo, para $t=0$, pode-se ter uma velocidade inicial, $v_{i}$, e para $t>0$ pode-se ter uma velocidade $v_{n}$, que representa a velocidade imediatamente anterior. Tomando para a condição inicial $v_{n}$, tem-se $v(0)=v_{n}$ e $A=v_{n}-\frac{F_{c}}{\xi_{B}}$, substituindo na equação (21) obtém:

$$
v(t)=\left(v_{n}-\frac{F_{c}}{\xi_{B}}\right) e^{\left(-\frac{\xi_{B}}{m_{B}}\right) t}+\frac{F_{c}}{\xi_{B}} .
$$

Sabendo que a velocidade da bola é dada pela Equação (22), a posição da bola pode ser obtida integrando essa equação em relação ao tempo. Logo,

$$
\begin{gathered}
p(\tau)=\int_{0}^{t} v(\tau) d \tau \\
p(\tau)=\int_{0}^{t}\left(\left(v_{n}-\frac{F_{c}}{\xi_{B}}\right) e^{\left(-\frac{\xi_{B}}{m_{B}}\right) \tau}+\frac{F_{c}}{\xi_{B}}\right) d \tau .
\end{gathered}
$$

A solução da Equação (24) é:

$$
p(t)=\left(v_{n}-\frac{F_{c}}{\xi_{B}}\right)\left(\frac{m_{B}}{\xi_{B}}\right)\left(1-e^{\left(-\frac{\xi_{B}}{m_{B}}\right) t}\right)+\frac{F_{c}}{\xi_{B}} t+C .
$$

A constante $C$ também pode ser determinada pela condição inicial do sistema, para cada instante de tempo tem-se uma posição para a bola, assim, no instante $t=0$ existe uma posição inicial $p_{i}$, e, para $t>0$ cada avanço na posição é realizado a partir de uma posição anterior $p_{n}$. Logo, tomando de forma genérica $p_{n}$, tem-se $p(0)=p_{n}$, assim, tem-se que $C=p_{n}$. Obtém-se então que:

$$
p(t)=p_{n}+\frac{F_{c}}{\xi_{B}} t+\left(v_{n}-\frac{F_{c}}{\xi_{B}}\right)\left(\frac{m_{B}}{\xi_{B}}\right)\left(1-e^{\left(-\frac{\xi_{B}}{m_{B}}\right) t}\right) .
$$

Retomando as componentes $x$ e $y$ para a velocidade e posição da bola tem-se:

$$
\begin{gathered}
v_{x}(t)=\left(v_{x n}-\frac{F_{c x}}{\xi_{B}}\right) e^{\left(-\frac{\xi_{B}}{m_{B}}\right) t}+\frac{F_{c x}}{\xi_{B}}, \\
v_{y}(t)=\left(v_{y n}-\frac{F_{c y}}{\xi_{B}}\right) e^{\left(-\frac{\xi_{B}}{m_{B}}\right) t}+\frac{F_{c y}}{\xi_{B}}, \\
p_{x}(t)=p_{x n}+\frac{F_{c x}}{\xi_{B}} t+\left(v_{n}-\frac{F_{c x}}{\xi_{B}}\right)\left(\frac{m_{B}}{\xi_{B}}\right)\left(1-e^{\left(-\frac{\xi_{B}}{m_{B}}\right) t}\right),
\end{gathered}
$$

$p_{y}(t)=p_{y n}+\frac{F_{c y}}{\xi_{B}} t+\left(v_{n}-\frac{F_{c y}}{\xi_{B}}\right)\left(\frac{m_{B}}{\xi_{B}}\right)\left(1-e^{\left(-\frac{\xi_{B}}{m_{B}}\right) t}\right)$.

Sabendo que o Filtro de Kalman é um processo iterativo e recursivo, e a trajetória é uma sucessão de posições, faz-se necessário o uso de equações recursivas para utilizar a modelagem apresentada nas etapas de predição e atualização do filtro. Logo, contemplando esses fatores, tem-se:

$$
\begin{gathered}
v_{x}(i)=\left(v_{x}(i-1)-\frac{F_{c x}(i)}{\xi_{B}}\right) e^{\left(-\frac{\xi_{B}}{m_{B}}\right) \Delta t}+\frac{F_{c x}(i)}{\xi_{B}}, \quad(31) \\
v_{y}(i)=\left(v_{y}(i-1)-\frac{F_{c y}(i)}{\xi_{B}}\right) e^{\left(-\frac{\xi_{B}}{m_{B}}\right) \Delta t}+\frac{F_{c y}(i)}{\xi_{B}},(32) \\
p_{x}(i)=p_{x}(i-1)+\frac{F_{c x}(i)}{\xi_{B}} \Delta t+\left(v_{x}(i-1)-\frac{F_{c x}(i)}{\xi_{B}}\right) \\
\times\left(\frac{m_{B}}{\xi_{B}}\right)\left(1-e^{\left(-\frac{\xi_{B}}{m_{B}}\right) \Delta t}\right),
\end{gathered}
$$

$$
\begin{aligned}
p_{y}(i)=p_{y}(i & -1)+\frac{F_{c y}(i)}{\xi_{B}} \Delta t+\left(v_{y}(i-1)-\frac{F_{c y}(i)}{\xi_{B}}\right) \\
& \times\left(\frac{m_{B}}{\xi_{B}}\right)\left(1-e^{\left(-\frac{\xi_{B}}{m_{B}}\right) \Delta t}\right) .
\end{aligned}
$$

As Equações (31), (32), (33) e (34) são equações nãolineares, dessa forma, para a previsão das posições da bola é necessário utilizar o Filtro de Kalman Estendido, de modo que essas equações são utilizadas na fase de predição e nos cálculos dos Jacobianos. 


\section{AQUISIÇÃO DE PARÂMETROS FÍSICOS}

De porte da modelagem da trajetória da bola, faz-se necessário determinar os parâmetros físicos que integram o modelo. De acordo com as regras da Robocup (Committee, 2018), a bola possui $44 \mathrm{~g}$ e diâmetro de $100 \mathrm{~mm}$.

Na documentação do robô NAO (Robotics, 2018) são disponibilizadas algumas informações sobre o motor da junta do NAO responsável pelo chute, este motor está localizado no tornozelo do NAO e denominado AnkleRoll. Estes dados estão na Tabela 1.

\section{Tabela 1. Especificação Motor}

\begin{tabular}{cc} 
Modelo & 22NT82213P \\
\hline Velocidade sem carga & $8300 \mathrm{rpm}$ \\
Torque nominal & $16,1 \mathrm{mNm}$ \\
Redução de velocidade & 201,3 \\
\hline
\end{tabular}

Como existe um sistema de redução de velocidade, o torque de saída é dado pela Equação (35) como explicado em (Garcia, 2005), da qual obtém-se que o torque de saída é $3,24093 \mathrm{Nm}$,

$$
\frac{T_{a}}{T_{b}}=\frac{r_{a}}{r_{b}}=\alpha
$$

A distância entre o eixo do motor e a ponta do pé do $\mathrm{NAO}$, a qual pode ser vista como um braço de alavanca, foi medida no robô e equivale a $13,5 \mathrm{~cm}$. A relação entre força e torque é discutida em (Garcia, 2005) e dada pela Equação (36), logo, a força nominal na ponta do pé que seria a força máxima do chute do NAO é $24 \mathrm{~N}$,

$$
T=F \times d \text {. }
$$

Ainda para caracterizar a força do chute, dada pela Equação 5, os parâmetros a, b e c foram obtidos de forma empírica, via testes de vídeo e simulações adequando-os ao perfil da força do chute. A constante $a$ representa o tempo de contato entre robô e bola, aproximadamente $0,3 \mathrm{~s}$. O $b$ define o caimento da curva, estipulado como 10. E $c$ é o centro da curva da função GBell, definido como 0, o parâmetro $t$ é o tempo de simulação.

Para cálculo do desvio padrão, os dados de movimento da bola foram obtidos pelo software Tracker e analisados no Matlab. A trajetória feita pela bola real, curva em linha tracejada em preto na Figura 6, e a trajetória teórica (sem erros), curva pontilhada em vermelho, são exibidas para análise. Dessa forma, calculou-se o desvio entre as duas trajetórias, caracterizando o desvio padrão da movimentação da bola e equivalente a 15,8939mm.

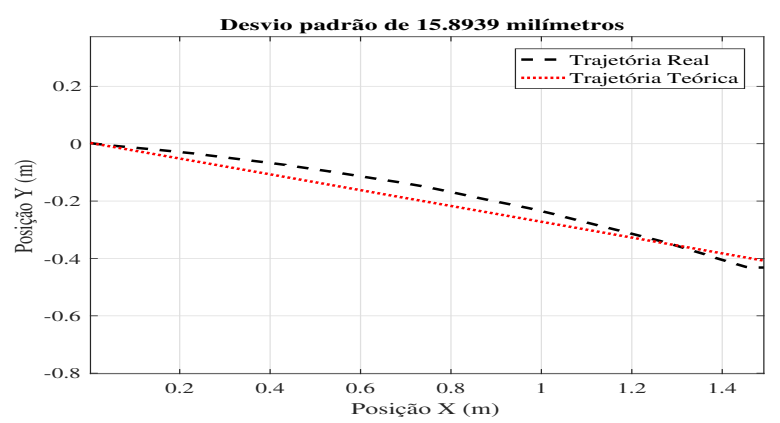

Figura 6. Cálculo do desvio Padrão da Trajetória da bola
Para cálculo do desvio padrão do sensor de captura de dados, a câmera, colocou-se a bola em uma posição conhecida enquanto o NAO a detectava. O desvio padrão foi calculado em relação a medida real de posicionamento da bola e as medidas aquisitadas. Os resultados dos testes e ensaios realizados estão dispostos na Tabela 2.

Para cálculo do coeficiente de atrito, tomou-se como cenário o robô chutando a bola no campo de carpete e mediuse a posição inicial, a posição final e o tempo desde que a bola é chutada até sua parada, substituiu-se os dados nas equações de movimento e obteve-se $0,1403178 \frac{\mathrm{Kg}}{\mathrm{s}}$. Por fim, os parâmetros de posições e velocidades iniciais foram arbitrados nas simulações.

Tabela 2. Desvio Padrão do sensor

\begin{tabular}{cc} 
Direção & Desvio Padrão $(\mathrm{mm})$ \\
\hline $\mathrm{X}$ & 5,53265 \\
$\mathrm{Y}$ & 2,39333 \\
\hline
\end{tabular}

\section{RESULTADOS}

Para validação do modelo proposto e dos parâmetros obtidos, e, tendo em mente o objetivo principal do trabalho, predizer as posições e velocidades da bola o Filtro de Kalman Estendido foi implementado no Matlab.

A Figura 7 mostra a trajetória realizada pela bola. A trajetória i) preta representa o comportamento real da bola, a ii) verde são as medidas aquistas pelo sensor, no caso, a câmera, e a iii) vermelha é a saída do Filtro de Kalman, iniciado em uma posição distante do início verdadeiro da bola. Como é possível observar, o filtro fornece uma boa estimativa das posições visto que até quando a posição do filtro é iniciada em uma posição longe da posição real da bola, o filtro consegue convergir para a posição real.

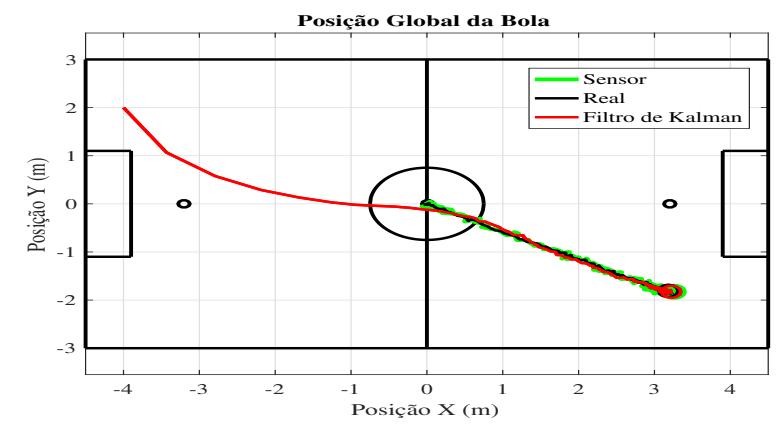

Figura 7. Predição da trajetória da bola com Filtro de Kalman Estendido.

Essa afirmativa pode ser confirmada na Figura 8 , a qual mostra que a diferença entre a posição real e a estimada tende a zero ao longo do processo.

O comportamento das posições em X e Y ao longo das iterações da simulação podem ser visualizada na Figura 9. A curva exibida se assemelha com as medições obtidas nos testes com o robô, relatadas na Figura 2.

O perfil da velocidade é mostrado na Figura 10. É possível notar que a velocidade aumenta muito rapidamente até um determinado patamar, que é caracterizado pela ação 

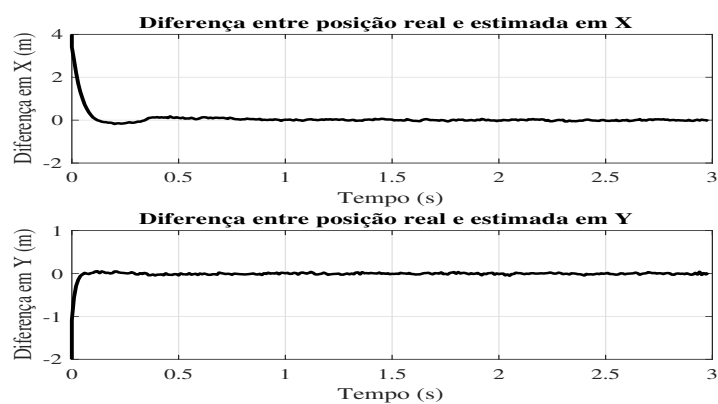

Figura 8. Diferença entre Posição Real e Estimada
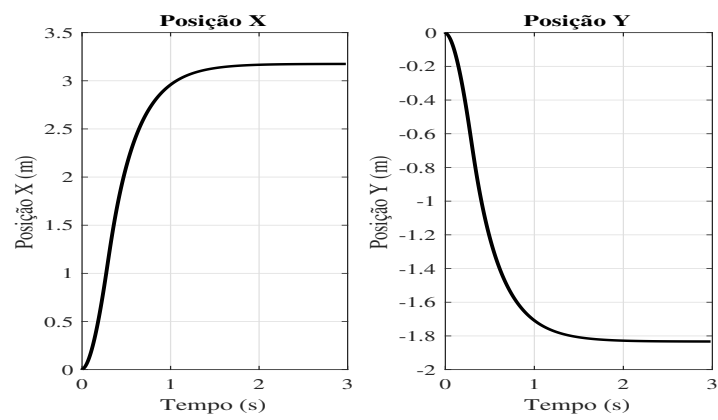

Figura 9. Posição X e Y

da força do chute do robô. Após cessar o contato do robô com a bola, a velocidade tende a decrescer até parar o movimento, pois a bola está sob a ação dos decréscimos produzidos pela força de atrito na força resultante. Assim, a força do chute impressa inicialmente é reduzida na resultante pelo atrito até a velocidade ser nula.
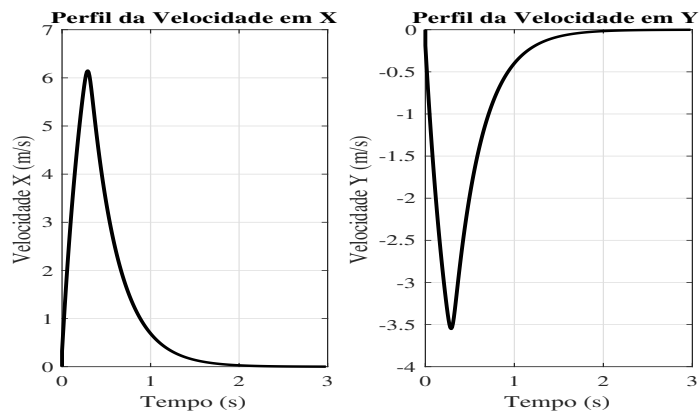

Figura 10. Velocidades X e Y

\section{CONCLUSÃO}

No presente trabalho foi realizada a predição da trajetória da bola para aplicação no futebol de robôs. Desenvolveu-se uma modelagem matemática diferente da usual apresentada na literatura para gerar o movimento da bola. Como ferramenta de filtragem estocástica utilizou-se o Filtro de Kalman Estendido para predizer as posições em X e Y da bola de futebol, bem como as respectivas velocidades.

Os ensaios em conjunto com as simulações indicaram que a modelagem desenvolvida neste trabalho possui acurácia com a mecânica de movimentos que ocorrem na realidade. Isto se comprova no Filtro de Kalman, pois este mostra-se bastante robusto para acompanhar a dinâmica da bola. Isto só é possível se o modelo matemático de predição for acurado, pois, mesmo quando a posição da bola dada pelo filtro é iniciada muito longe da posição real, o mesmo possui a capacidade de convergir para a posição real.

Durante o jogo, quando o robô está em movimento, perdas da detecção da bola são frequentes, dessa forma, com a predição da bola, o robô continua tendo uma estimativa da posição da bola nessas condições adversas. Assim, diversas novas estratégias de jogo podem ser desenvolvidas, enriquecendo a área.

\section{REFERÊNCIAS}

Amaral, E. (2015). Detecção e rastreamento de veículos em movimento para automóveis robóticos autônomos. Harpers.

Botta, A., Fabro, J., and Kaestner, C. (2013). Comparação entre dois modelos de redes neurais artificiais e máquinas de vetores suporte, aplicados à predição da posição da bola da categoria f180 da robocup. Proceeding Series of the Brazilian Society of Applied and Computational Mathematics, SBMAC 2013, 1, 1-6.

Bustamante, C., Flores, C., and Garrido, L. (2007). A physics model for the robocup 3d soccer simulation. Conference: Proceedings of the 2007 Spring Simulation Multiconference - SpringSim, 2, 151-158.

Committee, R.T. (2018). Robocup standard platform league (nao) rule book. https : //spl.robocup.org/wpcontent/uploads/downloads/Rules2018.pdf.

Garcia, C. (2005). Modelagem e Simulação de Processos Industriais e de Sistemas Eletromecânicos. Edusp, São Paulo.

Junior, F.R. (2015). Sistema de interceptação de bolas no futebol de robôs - small size league. Iniciação Científica - Departamento de Ciência da Computação, FEI.

Malheiro, D. (2010). Sistema de estratégia para posicionamento eficiente da defesa no futebol de robôs. Iniciação Científica - Laboratório de Robótica e Inteligência Artificial da FEI.

Montanari, R. (2015). Deteç̧ão e classificação de objetos em imagens para rastreamento de veículos. Ph.D. thesis, Universidade de São Paulo.

Motta, J. and Tourino, S. (2008). Sistema de rastreamento por visão em robôs móveis com otimização por projeto fatorial. Revista Iberoamericana de Ingeniería Mecánica, 12, 25-34.

Rivero, P. (2004). Técnicas de rastreamento e aplicações em análise cinemática de movimentos humanos. Ph.D. thesis, Universidade Estadual de Campinas.

Robotics, S. (2018). Nao software 1.14 .5 documentation. http : //doc.aldebaran.com/1 14/family/nao $25 /$ motor $_{h} 25$.html \#h25 - motors.

Röfer, T., Laue, T., Bülter, Y., Krause, D., Kuball, J., and Mühlenbrock, A. (2017). B-human team report and code release 2017. RoboCup 2017.

Simões, A., Costa, A., and Garcia, C. (2002). Regressão e predição de trajetórias: um comparativo das técnicas clássicas e sua aplicação para a visão computacional no domínio do futebol de robôs. Workshop de Computação - WORKCOMP'2002, 101-107.

Teh, B. (2011). Ball modelling and its applications in robot goalie behaviours. Ph.D. thesis, The University of New South Wales. 